\title{
Bosonic (meta)stabilization of cosmic string loops
}

\author{
J.R. Morris \\ Physics Dept., Indiana University Northwest, \\ 3400 Broadway, Gary, Indiana 46408 USA
}

\begin{abstract}
We consider the possibility of a bosonic (meta)stabilization of a cosmic gauge string loop due to the presence of a gas of low mass bosonic particles which become trapped within the string core. This boson gas exerts a pressure which tends to counteract the string tension, allowing a circular string loop to find a finite equilibrium radius, provided that gas particles do not escape the string core. However, high energy bosons do escape, and consequently the loop shrinks and the temperature rises. Estimates indicate that the bosonic stabilization mechanism is ineffective, and the loop is unstable against decay.
\end{abstract}

PACS numbers: 11.30.Qc, 11.17.+y, 98.80.Cq

Keywords: cosmic string loop, bosonic stabilization, domain kink

\section{INTRODUCTION}

The effects of fermions on the stability of nontopological defects, such as semilocal and electroweak strings[1],[2], has been studied with some interesting results. For example, the presence of fermions within the core of a nontopological string can have a stabilizing effect [3], [4], [5]. (See, e.g., [6] for an extensive review.) Presently, we investigate the possible existence of a bosonic stabilizing effect for Abelian-Higgs string loops.

In Ref.[7] a model was introduced where, by the Witten mechanism[8], a complex-valued gauge string scalar field $\chi$ interacts with a $\mathrm{U}(1)$ gauge field $A_{\mu}$ along with a real-valued scalar field $\phi$, instead of a complex scalar field. So, unlike the Witten superconducting string model, there is only one $\mathrm{U}(1)$ gauge field, confined to the string core, and the non-string scalar field $\phi$ is real rather than complex. It was seen in [7] that for a certain range of parameters a discrete $Z_{2}$ symmetry associated with the scalar $\phi$ could be spontaneously broken within the string core, resulting in a set of vacuum domains where $\phi= \pm \phi_{0}$. The $+\phi_{0}$ and $-\phi_{0}$ vacuua are separated by topological solitonic "kinks" and "antikinks" described by $\phi_{K}$ and $\phi_{\bar{K}}$ interpolating between the vacuum states $\pm \phi_{0}$. If the string is considered as a thin tube, the domain kinks can be thought of as small domain wall sections within the string core, and in the limit that the string is considered infinitely thin, the domain kinks are just ordinary kinks of an effective $1+1$ dimensional field theory in the string core.

The kinks $(\mathrm{K})$ and antikinks $(\overline{\mathrm{K}})$ will undergo annihilations and produce elementary $\varphi$ bosons, which are the perturbative particle excitations of the $\phi$ field. The $\varphi$ bosons can have a mass $m_{\text {in }}$ inside the string core which is much less than the $\varphi$ boson mass $m=m_{\text {out }}$ outside the string, where the vacuum state is $\phi=0$. Thus, assuming this to be the case, i.e., $m_{\text {in }} \ll m$, the $\varphi$ bosons will get effectively trapped within the string, with a slow rate of 
leakage associated with the escape of high energy $\varphi$ bosons with energies $E \gtrsim m$, if the $\varphi$ gas temperature remains low, $\beta m \gg 1$. (In Ref.[7] the oppsosite case $m_{i n} \gg m$ was assumed, resulting in $\varphi$ boson radiation being released into the vacuum outside the string.) However, if $m_{\text {in }} \ll m$, a mechanism can exist where the trapped $\varphi$ bosons create what we may call here a "pressurized" string, for lack of a better name. Closed string loops can form through intercommutations and self intersecting trajectories. The $\varphi$ pressure in a string loop leads to a tendency for the loop to expand, while the string tension tends to cause it to shrink. The result will be a stabilization at some equilibrium radius $\mathcal{R}$ and length $L=2 \pi \mathcal{R}$ for a circular string loop. This string loop may actually exist as a metastable state over longer periods of time, whose rate of decay ultimately depends upon the rate of energy loss through gravitational and $\chi$ boson radiation, as well as the rate of $\varphi$ particle leakage from the string, which, in turn, depends upon $m_{i n} / m$ and the $\varphi$ energy distribution within the string.

However, an analysis of a loop's stability against decay due to $\varphi$ gas loss indicates that although the $\varphi$ gas may leak out at a relatively slow rate at first, the rate rapidly increases due to an increasing temperature. The lifetime of this bosonic stabilization mechanism, treating the loop as a perfect $\phi$ blackbody for $\varphi$ particle energies $E \geq m$, is found to be $\tau \sim m_{\chi}^{-1}$, where $m_{\chi}$ is the $\chi$ boson mass. This time $\tau$ is very short in comparison to the string's lifetime due to gravitational radiation, and consequently the stabilization mechanism is found to be ineffective, so that the loop remains unstable against decay.

In section II a brief review of the field theory model describing kinks nested within a host string is given, with $\mathrm{K}-\overline{\mathrm{K}}$ annihilations contributing to a confined gas of $\mathrm{K}-\overline{\mathrm{K}}$ states and $\varphi$ bosons, producing a pressurized string. For $m_{i n} \ll M_{K}, \mathrm{~K}-\overline{\mathrm{K}}$ annihilations are much more probable than $\mathrm{K}-\overline{\mathrm{K}}$ pair productions from $\varphi$ bosons, and the gas within the string core therefore consists mostly of $\varphi$ bosons. The equilibrium conditions for a circular string loop are given in section III, and the process of loop decay is studied in section IV. Particle masses and model constraints are listed in section V. Section VI forms a brief summary.

\section{THE MODEL}

\section{A. Kinks nested within a gauge string}

The gauge string - Let's first consider a straight Abelian gauge string [9 11] lying on the $z$ axis. There is a complex-valued scalar string field $\chi=\frac{1}{\sqrt{2}} R e^{i \alpha}$ that interacts with the U(1) gauge field $A_{\mu}=\frac{1}{e}[P(\vec{x}, t)-1] \partial_{\mu} \alpha$ and also with a real-valued scalar field $\phi$. The Lagrangian for this system is

$$
L=\left(D^{\mu} \chi\right)^{\star}\left(D_{\mu} \chi\right)+\frac{1}{2} \partial^{\mu} \phi \partial_{\mu} \phi-V-\frac{1}{4} F^{\mu \nu} F_{\mu \nu},
$$

where the gauge covariant derivative $D_{\mu}$ and the $\mathrm{U}(1)$ field tensor $F_{\mu \nu}$ are

$$
D_{\mu}=\nabla_{\mu}+i e A_{\mu}, \quad F_{\mu \nu}=\partial_{\mu} A_{\nu}-\partial_{\nu} A_{\mu}=\frac{1}{e}\left(\partial_{\mu} P \partial_{\nu} \alpha-\partial_{\nu} P \partial_{\mu} \alpha\right),
$$


The potential $V$ for the system takes the form

$$
\begin{aligned}
V & =\lambda\left(\chi^{\star} \chi-\frac{1}{2} \eta^{2}\right)^{2}+f\left(\chi^{\star} \chi-\frac{1}{2} \eta^{2}\right) \phi^{2}+\frac{1}{2} m^{2} \phi^{2}+\frac{1}{4} g \phi^{4} \\
& =\frac{1}{4} \lambda\left(R^{2}-\eta^{2}\right)^{2}+\frac{1}{2} f\left(R^{2}-\eta^{2}\right) \phi^{2}+\frac{1}{2} m^{2} \phi^{2}+\frac{1}{4} g \phi^{4} .
\end{aligned}
$$

Here, $\nabla_{\mu}$ represents the ordinary spacetime covariant derivative, and a metric with signature $(+,-,-,-)$ is used. The coupling constants $\lambda, f$, and $g$, along with the $\phi$ particle mass $m$, are taken to be positive, real quantities. The stable vacuum state of the theory is located by $R=\eta, \phi=0$, and the $\chi$ particle mass is $m_{\chi}=(2 \lambda)^{\frac{1}{2}} \eta$.

From (1) the field equations for this system can be obtained:

$$
\begin{gathered}
\nabla_{\mu} \partial^{\mu} R-R P^{2} \partial_{\mu} \alpha \partial^{\mu} \alpha+\left[\lambda\left(R^{2}-\eta^{2}\right)+f \phi^{2}\right] R=0, \\
\nabla_{\mu}\left(\partial^{\mu} P \partial^{\nu} \alpha-\partial^{\nu} P \partial^{\mu} \alpha\right)+e^{2} R^{2} P \partial^{\nu} \alpha=0, \\
\nabla_{\mu} j^{\mu}=0, \quad j_{\mu}=-R^{2} P \partial_{\mu} \alpha \\
\nabla_{\mu} \partial^{\mu} \phi+\left[f\left(R^{2}-\eta^{2}\right)+m^{2}+g \phi^{2}\right] \phi=0 .
\end{gathered}
$$

Now, when the real scalar field $\phi=0$, this model possesses a solution set that describes an Abelian gauge string. With cylindrical coordinates $(r, \theta, z)$ a static string lying on the $z$ axis is depicted by the functions $R(r), P(r)$, and $\alpha=\theta$. Theses are subject to the boundary conditions $R(0)=0, P(0)=1$ in the center of the string core, and asymptotically, outside the string core, $R \rightarrow \eta, P \rightarrow 0$. The radius of the string is $r_{0} \approx m_{\chi}^{-1} \approx\left(2 \lambda \eta^{2}\right)^{-\frac{1}{2}}$. For mathematical convenience and simplicity, we consider the string as a tube of false vacuum of radius $r_{0}$ with $R \approx 0$ in the interior and $R \approx \eta$ outside.

However, when the scalar field $\phi$ does not vanish identically, there is a parameter range for which it becomes energetically favorable for a $\phi$ condensate to form within the string core[7]. To see this, set $R \approx 0$ in the string core, and then the potential $V$ given by (3) is minimized by a field configuration for which $\phi= \pm \phi_{0}$, where

$$
\phi_{0}=\left[\left(f \eta^{2}-m^{2}\right) / g\right]^{\frac{1}{2}}
$$

is a positive constant for $f \eta^{2}-m^{2}>0$. Then $V\left(0, \pm \phi_{0}\right)=\frac{1}{4}\left(\lambda \eta^{4}-g \phi_{0}^{4}\right)<V(0,0)=\frac{1}{4} \lambda \eta^{4}$. Of course the field $\phi$ has gradient energy also, owing to the fact that $\phi \rightarrow 0$ outside the string. As was shown in [7] there is a parameter range for which $\phi=0$ is unstable in the string core, and a condensate with $\phi= \pm \phi_{0}$ forms there, breaking the $Z_{2}$ symmetry, with $\phi \rightarrow 0$ outside the string. We will assume that the model parameters do indeed assume values that allow the formation of the $\phi= \pm \phi_{0}$ condensate in the core.

Kinks - We denote the static string background fields by $R_{s}$ and $P_{s}$ and adopt the ansatz $R \approx R_{s}(r), P \approx P_{s}(r), \alpha=\theta$, and $\phi=\phi(r, z, t)$. Then (7) becomes, approximately, 


$$
-\partial_{0}^{2} \phi+\partial_{r}^{2} \phi+\frac{1}{r} \partial_{r} \phi+\partial_{z}^{2} \phi-\left[g\left(\phi^{2}-\phi_{0}^{2}\right)+f R^{2}\right] \phi=0,
$$

with the boundary conditions $\phi \rightarrow \phi(z, t)$ as $r \rightarrow 0, \phi \rightarrow 0$ as $r \rightarrow \infty$, and $|\phi| \rightarrow \phi_{0}$ as $|z| \rightarrow \infty$. Using our tube representation for the string, we write

$$
R \approx\left\{\begin{array}{l}
0, r \leq r_{0} \\
\eta, r>r_{0}
\end{array}\right\}, \quad \phi \approx\left\{\begin{array}{cl}
\phi(z, t), & r \leq r_{0} \\
0, & r>r_{0}
\end{array}\right\}
$$

so that in the string core (9) simplifies to

$$
-\partial_{0}^{2} \phi+\partial_{z}^{2} \phi-g \phi\left(\phi^{2}-\phi_{0}^{2}\right)=0
$$

A static solution of (11) is given by

$$
\phi_{K}(z)=\phi_{0} \tanh \left(\frac{z-z_{0}}{w}\right), \quad w=\frac{1}{\phi_{0}}\left(\frac{2}{g}\right)^{\frac{1}{2}},
$$

representing a static domain kink $(\mathrm{K})$ of width $w$ located at the position $z=z_{0}$ in the string. A domain antikink $(\bar{K})$ solution [12] is given by $\phi_{\bar{K}}(z)=-\phi_{K}(z)$, and a time-dependent Lorentz boosted domain kink with velocity $u$ [12] is given by the solution

$$
\phi_{K}(z ; u)=\phi_{0} \tanh \left[\frac{\left(z-z_{0}\right)-u t}{w\left(1-u^{2}\right)^{\frac{1}{2}}}\right],
$$

The spatial gradient of a static kink or antikink solution $\phi$ is

$$
\partial_{z} \phi= \pm \sqrt{\frac{g}{2}}\left(\phi^{2}-\phi_{0}^{2}\right)= \pm \sqrt{\frac{g}{2}} \phi_{0}^{2} \operatorname{sech}^{2}\left(\frac{z-z_{0}}{w}\right)
$$

which rapidly vanishes for $\left|z-z_{0}\right| \gg w$, with $\phi_{K} \rightarrow \pm \phi_{0}$ as $\left(z-z_{0}\right) \rightarrow \pm \infty$.

\section{B. Kink formation}

Using the potential $V(\chi, \phi)$ given in (3), we set $\chi=0$ and define an effective potential $U(\phi)$ for the $\phi$ field [7] by subtracting off the constant $V(0,0)=\frac{1}{4} \lambda \eta^{4}$ :

$$
U(\phi)=V(0, \phi)-V(0,0)=V(0, \phi)-\frac{1}{4} \lambda \eta^{4}=\frac{1}{4} g \phi^{2}\left(\phi^{2}-2 \phi_{0}^{2}\right),
$$

This is then used to define an effective Lagrangian for the $\phi$ field,

$$
L^{(\phi)}=\frac{1}{2} \partial^{\mu} \phi \partial_{\mu} \phi-U(\phi) .
$$

which, in turn, can be used to define the energy-momentum tensor associated with the field $\phi$, 


$$
T_{\mu \nu}^{(\phi)}=\partial_{\mu} \phi \partial_{\nu} \phi-g_{\mu \nu} L^{(\phi)}
$$

(We can note here that the equation of motion (11) for the field $\phi(z, t)$ in the string core is obtained from the variation of $L^{(\phi)}$.) Therefore the energy density of the $\phi$ field in the string core is

$$
T_{00}^{(\phi)}=\frac{1}{2}\left[\left(\partial_{0} \phi\right)^{2}+\left(\partial_{z} \phi\right)^{2}\right]+U(\phi) .
$$

For a constant field configuration $\phi= \pm \phi_{0}$ we have that $T_{00}^{(\phi)}$ is negative, i.e., $T_{00}^{(\phi)}\left( \pm \phi_{0}\right)=$ $U\left(\phi_{0}\right)=-\frac{1}{4} g \phi_{0}^{4}$, but the total energy density in the string core (for $R \approx 0, A_{\mu} \approx 0, \phi= \pm \phi_{0}$ ) is approximately $T_{00}=T_{00}^{(\phi)}\left( \pm \phi_{0}\right)+\frac{1}{4} \lambda \eta^{4}=\frac{1}{4}\left(\lambda \eta^{4}-g \phi_{0}^{4}\right)$ which is positive, provided that $\lambda \eta^{4}-g \phi_{0}^{4}>0$, which is assumed to be the case.

Inside the string core, the lowest energy configuration for the $\phi$ field is $\phi= \pm \phi_{0}$, and the $\phi$ field will tend to settle into one of these states where either $\phi=+\phi_{0}$ or $\phi=-\phi_{0}$, but the domains of these different states will be uncorrelated beyond some coherence length $\xi$, which is expected to be at least as big as the width $w$ of a kink. Two different domains must be separated by a region where $\phi=0$, locating the center of a kink or antikink. We therefore take the average adjacent $\mathrm{K}-\overline{\mathrm{K}}$ separation distance to be $\xi \gtrsim w=\frac{1}{\phi_{0}}\left(\frac{2}{g}\right)^{\frac{1}{2}} \sim 1 /\left(\sqrt{g} \phi_{0}\right)$, and the solution $\phi_{K}$ (or $\phi_{\bar{K}}$ ) represents a kink (or antikink) which separates $\pm \phi_{0}$ domains. Two consecutive kinks are separated by an antikink, and vice versa, with an initial $\mathrm{K}-\overline{\mathrm{K}}$ separation distance of $\sim \xi$.

\section{Pressurized string}

The $\mathrm{K}$ and $\overline{\mathrm{K}}$ states are not expected to be static, but rather to move around, as described by (13), and consequently begin annihilating soon after formation. The $\mathrm{K}-\overline{\mathrm{K}}$ annihilations give rise to a gas of bosonic $\varphi$ particles in the string core. The $\varphi$ particles are excitations of the $\phi$ field above the ground state, i.e., $\phi=\phi_{0}+\varphi$, where the ground state condensate is $\phi_{0}$, say. We denote the mass of the $\varphi$ particles inside the core by $m_{i n}$. The $\varphi$ particle mass outside the core, in vacuum, is $m_{\text {out }}=m$ [see (3)]. For a large mass contrast where $m_{\text {in }} \ll m_{\text {out }}=m$, the $\varphi$ bosons are largely confined to the string core, although those particles with energies $E \gtrsim E_{\text {esc }}=m$ can eventually escape the core and reside in the vacuum. However, particles with energy $E<m$ are trapped inside the core. We assume here that there is indeed a high contrast in $\varphi$ particle masses inside and outside the string, such that $m_{\text {in }} \ll m_{\text {out }}=m$, and the entrapped bosons form a gas with temperature $T$ inside the string with a thermal energy distribution with $m / T=\beta m \gg 1$, so that according to Bose-Einstein statistics, where the number of particles of energy $E$ is $N(E)=\left(e^{\beta E}-1\right)^{-1}$, the rate at which $\varphi$ particles with $\beta E \gtrsim \beta m \gg 1$ escape is small. The bosonic gas exerts a pressure in the core which tends to counteract the effects of the string tension. We refer to the string whose core is filled with the $\varphi$ boson gas as a pressurized string. A closed loop of pressurized string can form through intercommutation processes or self-intersecting string trajectories. The pressurized string 
loop can be roughly thought of as resembling a (not too leaky) tire inner tube pressurized with air. Furthermore, we consider the case where the $\varphi$ gas is assumed to be relativistic, and can therefore have nonnegligible energy density $\rho$, number density $n$, and pressure $p$.

\section{LOOP STABILIZATION}

We contemplate a scenario where a loop of cosmic string forms with many $\mathrm{K}-\overline{\mathrm{K}}$ states in the core, which begin annihilating to produce a $\varphi$ boson gas within the string, with $m_{\text {in }} \ll m_{\text {out }}=m$, so that the bosons are effectively trapped within the string core and exert a pressure $p$. When the effect of the pressure, which tends to expand the loop, becomes comparable to that of the string tension, which tends to shrink the loop, an equilibrium can be reached at some loop length $L=2 \pi \mathcal{R}$ for a circular loop. During a shrinking process, energy is radiated away in the form of $\chi$ bosons and gravitational radiation. The equilibrium configuration minimizes the configuration energy $\mathcal{E}$, since the radial force on the loop is $F_{R}=-\delta \mathcal{E} / \delta \mathcal{R}=0$. (We do not consider a loop with a nonzero angular momentum of the boson gas.)

The energy of the loop near equilibrium is the sum of two terms,

$$
\mathcal{E}=\mu L+\rho_{G} A_{s} L
$$

where $\mu$ is the energy per unit length (tension) of the string (excluding the boson gas), $\rho_{G}$ is the energy density of the $\varphi$ gas, $A_{s}=\pi r_{0}^{2}$ is the cross sectional area of the string, and $L=2 \pi \mathcal{R}$ is the length of the circular loop with radius $\mathcal{R}$. We take the $\varphi$ bosons to be effectively massless, with the loop able to equilibrate at a temperature $m_{\text {in }} \ll T \ll m$. As a loop physically shrinks, energy is lost and the radial force $F_{R}<0$ until a physical equilibrium is reached. At that time, the loop energy is given by (19), with $L=$ const. This equilibrium value of $L$ is determined by minimizing $\mathcal{E}$ with respect to $L$. Care must be taken in this variation, however. We consider a virtual variation of the loop length $L$ while holding entropy $S$ and $\varphi$ boson particle number $N$ fixed. (One implies the other, since both are proportional to $T^{3} A_{s} L$.) Using $\rho_{G}=\frac{\pi^{2}}{30} T^{4}$ for the relativistic $\varphi$ boson gas, (19) becomes

$$
\mathcal{E}=\mu L+\left(\frac{\pi^{2}}{30} T^{4}\right) A_{s} L
$$

The entropy density of the gas is [13] $s=\frac{2 \pi^{2}}{45} T^{3}$ and the number density is[13] $n=\frac{\zeta(3)}{\pi^{2}} T^{3}$, so that holding entropy $S=s A_{s} L$ and particle number $N=n A_{s} L$ constant during the virtual variation of $L$ results in the equilibrium constraint $T^{3} L=$ const:

$$
\frac{N}{A_{s}}=\frac{\zeta(3)}{\pi^{2}} T^{3} L=c_{1} ; \quad \frac{S}{A_{s}}=\frac{2 \pi^{2}}{45} T^{3} L=c_{2} ; \quad T^{3} L=\frac{N \pi^{2}}{\zeta(3) A_{s}}=\frac{45 S}{2 \pi^{2} A_{s}} \equiv C
$$

Therefore, at equilibrium, the entropy per $\varphi$ particle is

$$
\frac{S}{N}=\frac{s}{n}=\frac{c_{2}}{c_{1}}=\frac{2 \pi^{4}}{45 \zeta(3)} \approx 3.6
$$


and from (21)

$$
T=C^{1 / 3} L^{-1 / 3}
$$

Using (23) in (20) gives an equilibrium loop energy

$$
\begin{aligned}
\mathcal{E} & =\mu L+\frac{\pi^{2}}{30} A_{s}\left(T^{3} L\right) T=\mu L+\frac{\pi^{2}}{30} C A_{s}\left(C^{1 / 3} L^{-1 / 3}\right) \\
& =\mu L+\frac{\pi^{2} A_{s} C^{4 / 3}}{30} L^{-1 / 3}=\mu L+C_{1} L^{-1 / 3}
\end{aligned}
$$

where $C_{1}=\pi^{2} A_{s} C^{4 / 3} / 30$. Minimizing this with respect to $L$ results in

$$
\frac{\partial \mathcal{E}}{\partial L}=\mu-\frac{1}{3} C_{1} L^{-4 / 3}=0 \Longrightarrow L=\left(\frac{C_{1}}{3 \mu}\right)^{3 / 4}, \quad L^{1 / 3}=\left(\frac{C_{1}}{3 \mu}\right)^{1 / 4}
$$

The equilibrium configuration energy is then

$$
\mathcal{E}=\mu\left(\frac{C_{1}}{3 \mu}\right)^{3 / 4}+C_{1}\left(\frac{3 \mu}{C_{1}}\right)^{1 / 4}=\left(C_{1}^{3} \mu\right)^{1 / 4}\left[\left(\frac{1}{3}\right)^{3 / 4}+3^{1 / 4}\right] \approx 1.75\left(C_{1}^{3} \mu\right)^{1 / 4}
$$

The gas energy to string energy ratio is

$$
\frac{\mathcal{E}_{G}}{\mathcal{E}_{s}}=\frac{3^{1 / 4}}{\left(\frac{1}{3}\right)^{3 / 4}}=3
$$

Therefore, the energy of the equilibrated loop is

$$
\mathcal{E}=\mathcal{E}_{s}+\mathcal{E}_{G}=4 \mathcal{E}_{s}=\frac{4}{3} \mathcal{E}_{G}=4 \mu L=4 \mu^{1 / 4}\left(\frac{1}{3} C_{1}\right)^{3 / 4} \propto \mu^{1 / 4} N
$$

From (21) the loop size is given by $L=C / T^{3}$, and since $\mathcal{E}=4 \mu L=8 \pi \mu \mathcal{R}$, the loop radius is

$$
\mathcal{R}=\frac{C}{2 \pi T^{3}}=\frac{\mathcal{E}}{8 \pi \mu}
$$

The Schwarzschild radius is $R_{S}=2 G \mathcal{E}=2 \mathcal{E} / M_{P}^{2}$, so

$$
\frac{\mathcal{R}}{\mathcal{R}_{S}}=\frac{1}{16 \pi G \mu} ; \quad \frac{\mathcal{R}}{\mathcal{R}_{S}}>1 \Longrightarrow G \mu<\frac{1}{16 \pi}
$$

The condition that the equilibrated loop radius lies outside the Schwarzschild radius manifests itself as an upper bound on the string tension, $G \mu<1 / 16 \pi$. This upper bound lies well above present observational constraints for the string tension, with $G \mu \lesssim 6.4 \times 10^{-7}$ for Abelian-Higgs strings [14], and therefore does not exclude any of the observational parameter space for the string tension. 
Now suppose that the loop is created with an initial radius less than the equilibrium radius, $\mathcal{R}_{0}<\mathcal{R}$. What happens in this case? Again, consider a virtual change in radius $\mathcal{R}_{0}$ while holding $N_{0}, S_{0}$ fixed, so that again we have the instantaneous constraint in (23) for the virtual deformation. Then by (24) we have

$$
\mathcal{E}_{0}=\mu L_{0}+C_{1} L_{0}^{-1 / 3}
$$

Now the radial components of the string tension and gas pressure forces, $F_{R, 0}^{s}$ and $F_{R, 0}^{G}$, respectively, are

$$
F_{R, 0}^{s}=-\frac{\partial \mathcal{E}_{0, s}}{\partial \mathcal{R}_{0}}=-2 \pi \mu ; \quad F_{R, 0}^{G}=-\frac{\partial \mathcal{E}_{0, G}}{\partial \mathcal{R}_{0}}=+\frac{1}{3}(2 \pi)^{-1 / 3} C_{1} \mathcal{R}_{0}^{-4 / 3}
$$

If $\left|F_{R, 0}^{G} / F_{R, 0}^{s}\right|>1$, the loop will expand, assuming, of course, $\mathcal{R}_{0}>\mathcal{R}_{S}$, and gas energy is converted into string energy in the process until $\left|F_{R}^{G} / F_{R}^{s}\right|=1$ at equilibrium. If $\left|F_{R, 0}^{G} / F_{R, 0}^{s}\right|<$ 1 , the loop shrinks, emits $\chi$ boson and gravitational radiation until it falls through its Schwarzschild radius, ending up as a black hole.

We see from (24) and (25) that the equilibrium length $L$ determined there corresponds to a point of stable equilibrium, so that for a dynamical loop with time varying radius, the loop radius will oscillate about the static eqilibrium value (assuming no loss of $\varphi$ bosons), implying short term stability. However, in the longer term loop energy will be lost due to a leakage of high energy $\varphi$ bosons, along with $\chi$ boson and gravitational radiation, leading to eventual loop decay. As long as the temperature is low enough, we might expect the loop to lose $\varphi$ bosons at only a slow rate, and the loop may stay fairly close to equilibrium (although $\chi$ boson and gravitational radiation are expected due to loop oscillations). We may argue that the $\varphi$ boson gas prefers to remain inside the string core in the form of $N \varphi$ bosons rather than outside the loop in the form of $N \phi$ bosons. This is because (provided the temperature $T$ is not too high) the pressurized string loop will have a configuration energy $\mathcal{E}$ which will be less than that of an unpressurized string loop with mass $\mathcal{E}_{s}$, plus $N \phi$ bosons (each of mass $m$ in vacuum outside the loop) with a minimal total energy of $\mathcal{E}_{s}+N m$. Specifically, we require $\mathcal{E}_{G}<N m$ for stability against a complete, rapid evaporation of the $\varphi$ gas. This translates into the condition

$$
\frac{\mathcal{E}_{G}}{N m}=\frac{B}{D} \frac{N T}{N m}=\frac{B}{D} \frac{T}{m}<1 ; \quad T<\frac{D}{B} m=.37 m
$$

where $N=\frac{\zeta(3)}{\pi^{2}} T^{3} A_{s} L \equiv D T^{3} L$ and $\mathcal{E}_{G}=\left(\frac{\pi^{2}}{30} T^{4}\right) A_{s} L \equiv B T^{4} L$ and we define

$$
B=\frac{\pi^{2} A_{s}}{30}, \quad D=\frac{\zeta(3) A_{s}}{\pi^{2}}, \quad \frac{D}{B}=\frac{30 \zeta(3)}{\pi^{4}}=.37
$$

Therefore, at low temperatures $T \ll m$ there may be a slow leakage of $\varphi$ bosons, but at higher temperatures we expect a departure from equilibrium, with a higher leakage rate. 


\section{LOOP DECAY}

Even with a stabilization mechanism as described above, we expect a loop to become unstable over longer periods of time. This is because a string loop can lose energy through the escape of high energy $\varphi$ particles of mass $m_{\text {in }}$ into the vacuum outside the string, where they become $\phi$ particles with mass $m$. An oscillating loop will also lose energy due to gravitational radiation [16] at a rate $P_{\text {rad }} \sim \gamma G \mu^{2}$, where $\gamma$ depends on the string trajectory, with $\gamma \sim 100$ being typical. The oscillating loop may also lose energy through $\chi$ boson radiation, but the $\chi$ boson radiation must come from nontraveling waves in the string[17]. (A string can, however, support certain traveling waves of arbitrary amplitude with no $\chi$ boson radiation[18].)

Let us focus here on the process of loop decay through $\phi$ boson radiation. For a simplified and qualitative approach, it is assumed that a $\varphi$ particle in the string gas with energy $E \geq m$ can exit the string and appear as a $\phi$ particle in the vacuum. A loop initially near equilibrium with mass $\mathcal{E}_{0}$ and $\varphi$ number $N_{0}$ at some initial time $t_{0}$ will eventually lose energy through $\phi$, $\chi$, and gravitational radiation, so that at a later time $t$ the loop mass is $\mathcal{E}$ and the $\varphi$ number is $N$. With the simple assumption that the number $n_{\phi}$ of $\phi$ particles produced in the time interval $t-t_{0}$ is given by $n_{\phi}=N_{0}-N$, we can write $\mathcal{E}_{0}=\mathcal{E}+n_{\phi} m+\mathcal{E}_{X}=\mathcal{E}+\left(N_{0}-N\right) m+\mathcal{E}_{X}$, where $\mathcal{E}_{X}$ represents $\phi$ kinetic energy, along with $\chi$ and gravitational energy that is produced by the decaying loop. We rewrite this as

$$
m N-\mathcal{E}=K+\mathcal{E}_{X} \equiv Q(t)
$$

where the constant $K=m N_{0}-\mathcal{E}_{0}$ and the function $Q(t)=K+\mathcal{E}_{X}(t)$ is a monotonically increasing function of time. (We note that the constant $K$ depends upon the initial loop mass and $\varphi$ particle number, i.e., the loop parameters near the equilibrium state, and we therefore do not regard $K$ as a universal constant for all loops in equilibrium. Each loop has its own value for $K$.) From (20), (21), and (34) we have

$$
\mathcal{E}=\left[\mu+\left(\frac{\pi^{2}}{30} T^{4}\right) A_{s}\right] L \equiv\left(\mu+B T^{4}\right) L, \quad N=\frac{\zeta(3) A_{s}}{\pi^{2}} T^{3} L \equiv D T^{3} L
$$

where

$$
B=\frac{\pi^{2} A_{s}}{30}, \quad D=\frac{\zeta(3) A_{s}}{\pi^{2}}, \quad \frac{B}{D}=2.7, \quad \frac{D}{B}=.37
$$

The condition (35) can now be written as

$$
m D T^{3}-B T^{4}=\mu+\frac{Q}{L}
$$

For a temperature $T \ll m$, the first term on the left hand side of (38) dominates, so that

$$
T \approx\left[\frac{1}{m D}\left(\mu+\frac{Q}{L}\right)\right]^{1 / 3}, \quad(T \ll m)
$$


As the loop evolves, it loses energy and shrinks and the loop temperature $T$ increases. (The constant $K$, and therefore $Q$, is positive. This can be seen from (36) and (28), since $\mathcal{E}_{0} \approx \frac{4}{3} \mathcal{E}_{G}=\frac{4}{3} B T^{4} L_{0} \ll N_{0} m$ for $T \ll m$.)

However, as $T$ increases (38) must break down beyond some value $T_{m}$, where our simple assumptions apparently begin to fail. One can see that this is the case by noticing that as $L$ decreases and $T$ increases, the right hand side (RHS) of (38) is monotonically increasing, but the left hand side (LHS) begins to decrease for $T>T_{m}$. The temperature $T_{m}$ therefore locates the local maximum of the LHS, which is found to occur at $T_{m}=\frac{3 D}{4 B} m \approx \frac{1}{4} m$. Therefore, the temperature $T$ and loop length $L$ are approximately related by (38) for $T \lesssim T_{m} \approx \frac{1}{4} m$, where we expect a more rapid rate of production of $\phi$ bosons to begin occurring. This value of $T_{m}$ is close to the temperature found in (33) where the loop begins to lose its bosonic stabilization.

For the loop near equilibrium with mass $\mathcal{E}_{0}$ and temperature $T_{0}$, we have $\mathcal{E}_{0}=4 \mu L_{0}=$ $\frac{4}{3} B T_{0}^{4} L_{0}$ which gives

$$
T_{0}=\left(\frac{3 \mu}{B}\right)^{1 / 4}=\left(\frac{90 \mu}{\pi^{3} r_{0}^{2}}\right)^{1 / 4} \sim\left(\mu m_{\chi}^{2}\right)^{1 / 4}=\left(G \mu M_{P}^{2} m_{\chi}^{2}\right)^{1 / 4} \lesssim 3 \times 10^{-2}\left(M_{P} m_{\chi}\right)^{1 / 2}
$$

where the Planck mass is $M_{P}=1 / \sqrt{G}$ and $G \mu \lesssim 6.7 \times 10^{-7}$ has been used[14]. This temperature is independent of loop size. Therefore all loops approach an equilibration at this temperature.

We then form the following qualitative picture of loop decay. A metastable loop begins to slowly shrink, and as it shrinks the temperature rises in accordance with (39), as long as $T \ll m$. As the temperature rises, the rate of $\phi$ boson emission increases and the rate of decay increases. However, our approximations and assumptions leading to (39) are expected to break down as $T$ approaches $m$, and the loop is expected to depart from being near equilibrium with a rapid release of $\phi$ radiation, along with $\chi$ particle and gravitational radiation.

We would like to obtain a rough estimate of how long the bosonic stabilization mechanism might persist. In order to do so, we treat the loop as a $\phi$ blackbody that emits $\varphi$ particles having energy $E \geq m$. The $\varphi$ particles are taken to be effectively massless. The energy density of $\varphi$ particles above this energy threshold is [15]

$$
u(T)=\frac{T^{4}}{2 \pi^{2}} \int_{\beta m}^{\infty} \frac{\eta^{3}}{e^{\eta}-1} d \eta=\frac{I(\beta m)}{2 \pi^{2}} T^{4}
$$

where $\eta=\beta E$. (This is $1 / 2$ the corresponding photon energy density, since photons have two polarization states.) For temperatures $T \ll m$, i.e., $\beta m \gg 1$,

$$
I(\beta m) \approx \int_{\beta m}^{\infty} \eta^{3} e^{-\eta} d \eta \approx(\beta m)^{3} e^{-\beta m} \ll 1, \quad(\beta m \gg 1)
$$


On the other hand, for a temperature $T_{*}=.37 m$, i.e., $\beta_{*} m=2.7$, as given in (33), we have

$$
I(2.7)=\int_{2.7}^{\infty} \frac{\eta^{3}}{e^{\eta}-1} d \eta \approx 4.4
$$

Therefore, for the $\varphi$ boson gas temperatures of interest we take, roughly, $I(\beta m) \lesssim 1$. The radiated power of $\phi$ bosons per unit area of the loop is [15]

$$
\mathcal{P}_{\phi}(T)=\frac{1}{4} u(T)=\frac{1}{A_{\text {loop }}} \frac{d \mathcal{E}}{d t} \lesssim \frac{T^{4}}{8 \pi^{2}}
$$

To obtain a very rough estimate for the lifetime of the bosonic stabilization mechanism to work, we set $\mathcal{E} \sim \mathcal{E}_{0} \approx \frac{4}{3} B T_{0}^{4} L_{0}, A_{\text {loop }} \sim 2 \pi r_{0} L_{0}$, and $\mathcal{P}_{\phi} \sim d \mathcal{E} / d t \sim \mathcal{E}_{0} / \tau$. This results in

$$
\tau \sim \frac{\mathcal{E}_{0}}{\mathcal{P}_{\phi} A_{\text {loop }}} \sim \frac{2 B T_{0}^{4}}{3 \pi r_{0}} \cdot \frac{8 \pi^{2}}{T_{0}^{4}} \sim \frac{16 \pi B}{3 r_{0}} \sim \frac{16 \pi}{3 r_{0}} \cdot \frac{\pi^{3} r_{0}^{2}}{30} \sim \frac{8 \pi^{4}}{45} m_{\chi}^{-1}
$$

where $r_{0} \sim m_{\chi}^{-1}$ has been used along with the definition of $B$ in (34). This lifetime is independent of the initial loop size $L_{0}$. This lifetime would be on the order of $10^{-14}$ seconds for $m_{\chi} \sim 1 \mathrm{eV}$, on the order of $10^{-23}$ seconds for $m_{\chi} \sim 1 \mathrm{GeV}$, and even smaller for a more massive $\chi$ boson.

An ordinary oscillating loop loses energy through gravitational radiation [16] at a rate $P_{\text {rad }} \sim \gamma G \mu^{2}$. Setting this equal to $\mathcal{E}_{0} / \Delta t \sim 4 \mu L_{0} / \Delta t$ gives $\Delta t \sim 4 L_{0} /(\gamma G \mu)$ as the lifetime of a loop due to gravitational energy loss. A comparison of $\tau$ and $\Delta t$ gives

$$
\frac{\tau}{\Delta t} \sim \frac{2 \pi^{4}}{45} \frac{\gamma G \mu}{m_{\chi} L_{0}} \sim \frac{2 \pi^{4}(\gamma G \mu)}{45} \frac{r_{0}}{L_{0}} \sim 4 \gamma G \mu \frac{r_{0}}{L_{0}} \lesssim 10^{-4} \frac{r_{0}}{L_{0}}
$$

where $r_{0} / L_{0}<1$ and $G \mu \lesssim 6.4 \times 10^{-7}$ has been used[14], along with $\gamma \sim 10^{2}$. It therefore appears that the bosonic stabilizing mechanism is effective for much less than one ten thousandth of the lifetime of the loop. The loop evidently loses its $\varphi$ boson gas long before decaying away.

We can notice that when we set the lower limit of the integral in (41) to zero, we obtain $I(0)=\pi^{4} / 15 \approx 6.5$, and in this case $u(T)=\rho_{G}$. An examination of (42) and (43) shows that initially, for the loop near the equilibrium state, we have $u / \rho_{G} \ll 1$, but at $T \sim T_{*}=.37 \mathrm{~m}$ we have $u / \rho_{G} \sim 2 / 3$. So initially only a small fraction of the $\varphi$ gas can escape the string core, but the temperature rapidly increases and at $T \sim T_{*}$ most of the gas can escape.

\section{PARTICLE MASSES AND MODEL PARAMETERS}

Let us calculate the $\varphi$ boson mass $m_{i n}$ and compare it to the kink mass $M_{K}$. From (15) and (16), with $R=0$ in the string core, and with $\phi=\phi_{0}+\varphi$,

$$
U(\phi)=\frac{1}{4} g \phi^{2}\left(\phi^{2}-2 \phi_{0}^{2}\right)=\frac{1}{4} g\left(\phi^{4}-2 \phi_{0}^{2} \phi^{2}\right)
$$


Then $m_{i n}^{2}=\partial^{2} U /\left.\partial \varphi^{2}\right|_{\varphi=0}=\partial^{2} U /\left.\partial \phi^{2}\right|_{\phi=\phi_{0}}=2 g \phi_{0}^{2}$. Using (8) for the definition of $\phi_{0}$ gives

$$
m_{i n}=\sqrt{2 g} \phi_{0}=\sqrt{2\left(f \eta^{2}-m^{2}\right)}
$$

For a large mass contrast $m_{i n} / m \ll 1$, we require

$$
2\left(f \frac{\eta^{2}}{m^{2}}-1\right) \ll 1, \quad \text { i.e., } \quad 2 g \frac{\phi_{0}^{2}}{m^{2}} \ll 1
$$

i.e., $\sqrt{g} \phi_{0} \ll m$, which can be satisfied for $m^{2} \sim O\left(f \eta^{2}\right)$, with $m^{2}<f \eta^{2}$.

The mass of a kink is[7]

$$
M_{K} \sim \frac{\sqrt{g} \phi_{0}^{3}}{\lambda \eta^{2}}
$$

This is obtained from the energy density $T_{00}^{(K)}$ of a static kink, which in turn is obtained from the energy density of the $\phi$ field in excess of that due to the condensate $\phi=\phi_{0}$ for a string containing only the condensate and kinks and antikinks:

$$
T_{00}^{(K)} \equiv T_{00}^{(\phi)}\left(\phi_{K}\right)-T_{00}^{(\phi)}\left( \pm \phi_{0}\right)=\left(\partial_{z} \phi_{K}\right)^{2}=\frac{1}{2} g \phi_{0}^{4} \operatorname{sech}^{4}\left(\frac{z-z_{0}}{w}\right)
$$

(This corrects a typo in Eq.(22) of Ref.[7].) An integration of $T_{00}^{(K)}$ over $z$ gives the kink surface energy density $\Sigma_{K}=\frac{4}{3} \sqrt{\frac{g}{2}} \phi_{0}^{3}$. (This corrects Eq.(23) of Ref.[7].) The kink mass is then $M_{K} \approx \Sigma_{K} r_{0}^{2}$, where $r_{0} \approx m_{\chi}^{-1}=\left(2 \lambda \eta^{2}\right)^{-1 / 2}$ is the radius of the string itself. The result is (50).

Finally, for the K, $\overline{\mathrm{K}}$ particles to be much heavier than the $\varphi$ particles in the string, so that $\overline{\mathrm{K} K}$ annihilations producing $\varphi$ bosons will dominate over the reverse where $\overline{\mathrm{K}} \mathrm{K}$ pairs are formed, we require

$$
\frac{m_{i n}}{M_{K}} \sim \frac{\lambda \eta^{2}}{\phi_{0}^{2}} \ll 1, \quad \text { or } \quad \lambda \ll \frac{\phi_{0}^{2}}{\eta^{2}}
$$

where (48) and (50) have been used. Since $m_{\chi}^{2} \sim \lambda \eta^{2}$, the above result can also be written as $\phi_{0}^{2} \gg m_{\chi}^{2}$. If (52) is not obeyed, we expect the string core to contain a mixed gas of kinks and $\varphi$ bosons. The gas is again treated as radiation as long as both the kink and $\varphi$ masses are small compared to the temperature $T$, i.e., $\beta M_{K} \ll 1$ and $\beta m_{i n} \ll 1$, so the basic mechanism for stabilization of a loop would still apply.

Some of the parameters and constraints for the model are summarized below.

$$
\begin{aligned}
& m_{\chi}=\sqrt{2 \lambda} \eta ; m_{\text {out }}=m ; \quad m_{\text {in }}=\sqrt{2 g} \phi_{0} ; \quad M_{K} \sim \frac{\sqrt{g} \phi_{0}^{3}}{\lambda \eta^{2}} \\
& \phi_{0}=\sqrt{\left(f \eta^{2}-m^{2}\right) / g} ; \quad w \sim \frac{1}{\sqrt{g} \phi_{0}}, \quad \xi \gtrsim w \\
& \frac{m_{\text {in }}}{m} \ll 1 \Longrightarrow \sqrt{g} \phi_{0} \ll m \Longrightarrow \sqrt{\left(f \eta^{2}-m^{2}\right)} \ll m \\
& T_{00}>0 \Longrightarrow \sqrt{\lambda} \eta^{2}>\sqrt{g} \phi_{0}^{2}: m_{\chi}^{2} / \sqrt{\lambda}>m_{\text {in }}^{2} / \sqrt{g}
\end{aligned}
$$




\section{SUMMARY}

A model of an Abelian-Higgs gauge string has been considered where there is an additional interaction of the scalar string field $(\chi)$ with a real-valued scalar field $(\phi)$. There exists a range of parameters for the model for which a $\phi$ condensate will tend to form in the string's core, settling into the low energy states $\phi= \pm \phi_{0}$, breaking a $Z_{2}$ symmetry. However, $+\phi_{0}$ and $-\phi_{0}$ domains will be uncorrelated beyond some coherence length $\xi$, and there will be regions where $\phi=0$ in between different domains. These $\phi=0$ regions locate kinks and antikinks that reside within the string core.

The kinks and antikinks will collide and undergo annihilations, producing $\varphi$ bosons, which are excitations of the $\phi$ field within the string core, $\phi=\phi_{0}+\varphi$, for instance. The mass $m_{\text {in }}$ of the $\varphi$ particles inside the string is not the same as the mass $m$ of the $\phi$ particles outside the string (where the vacuum state is located by $\phi=0$ ). The case considered here is that for which $m_{i n} \ll m$ and $m_{i n} \ll M_{K}$, where $M_{K}$ is the kink mass. In this case, $\varphi$ bosons are produced from $\mathrm{K}-\overline{\mathrm{K}}$ annihilations, and get trapped within the string core, giving rise to a gas pressure and additional energy. The gas pressure tends to cause the expansion of a closed string loop, while the string tension tends to cause a contraction. An equilibrium is established when these two effects are balanced and the configuration energy is minimized, provided that no $\varphi$ particles exit the string core. The equilibrium loop radius $\mathcal{R}$ is found to be given by the ratio of the string mass $\mathcal{E}$ and the string tension $\mu$, i.e., $\mathcal{R}=\mathcal{E} /(8 \pi \mu)=C /\left(2 \pi T^{3}\right)$ [see Eq.(29)] ], where $C$ is a constant depending on the number $N$ of $\varphi$ particles in the string loop.

However, high energy $\varphi$ bosons with energies $E \gtrsim m$ will escape the string core, so that the string loop is unstable over longer periods of time. The loop begins to shrink, relatively slowly at first, but at a rapidly increasing rate. An oscillating loop will also lose energy due to gravitational radiation [16] at a rate $P_{\text {rad }} \sim \gamma G \mu^{2}$, where $\gamma$ depends on the string trajectory, with $\gamma \sim 100$ being typical. The oscillating loop can also lose energy through $\chi$ boson radiation due to loop oscillations, but the $\chi$ boson radiation must come from nontraveling waves (e.g., standing waves) in the string[17]. (A string can, however, support certain traveling waves of arbitrary amplitude with no $\chi$ boson radiation[18].) However, estimates based upon the rate of $\phi$ particle emission by the loop indicate that the bosonic stabilization mechanism considered here is rather ineffective, lasting for a short duration which is much less than the lifetime of the loop due to gravitational radiation.

Acknowledgment: I thank an anonymous referee for valuable comments.

[1] T. Vachaspati and A. Achúcarro, Phys. Rev. D44, 3067 (1991)

[2] T. Vachaspati, Phys. Rev. Lett. 68, 1977 (1992); Erratum-ibid. 69, 216 (1992)

[3] D. Stojkovic, Int. J. Mod. Phys. A16S1C 1034-1036 (2001) \{hep-th/0103216\}

[4] G.D. Starkman, D. Stojkovic, and T. Vachaspati, Phys. Rev. D63, 085011 (2001) \{hep$\mathrm{ph} / 0007071\}$ 
[5] G. Starkman, D. Stojkovic, T. Vachaspati, Phys. Rev. D65, 065003 (2002) \{hep-th/0103039\}

[6] A. Achucarro and T. Vachaspati, Phys. Rept. 327, 347-426 (2000), Phys.Rept. 327 427(2000) $\{$ hep-ph/9904229\}

[7] J.R. Morris, Phys. Rev. D51, 697-702 (1995)

[8] E. Witten, Nucl. Phys. B 249, 557 (1985)

[9] H. B. Nielsen and P. Olesen, Nucl. Phys. B 61, 45 (1973)

[10] A. Vilenkin, Phys. Rep. 121, 263 (1985)

[11] A. Vilenkin and E.P.S. Shellard, Cosmic Strings and Other Topological Defects (Cambridge University Press, 1994)

[12] See, for example, R. Rajaraman, Solitons and Instantons (North-Holland Publishing Co., 1982)

[13] See, for example, E.W. Kolb and M.S. Turner, The Early Universe (Addison-Wesley, 1990)

[14] See, for example, R. Battye and A. Moss, Phys. Rev. D82, 023521 (2010) and references therein.

[15] See, for example, F. Reif, Fundamentals of Statistical and Thermal Physics, Chapter 9 (McGraw-Hill, 1965)

[16] B. Allen and P. Casper, Phys. Rev. D50, 2496 (1994)

[17] T. Vachaspati, A.E. Everett, and A. Vilenkin, Phys. Rev. D30, 2046 (1984)

[18] Vachaspati and T. Vachaspati, Phys. Lett. B238, 41-44 (1990) 